\title{
Tissue-specific inactivation of p53 tumor suppression in the mouse
}

\author{
Tammy Bowman, ${ }^{1}$ Holly Symonds, ${ }^{1,2}$ Liya Gu, ${ }^{1,3}$ Chaoying Yin, ${ }^{1}$ Moshe Oren, ${ }^{4}$ \\ and Terry Van Dyke \\ ${ }^{1}$ Department of Biochemistry and Biophysics, University of North Carolina at Chapel Hill, Chapel Hill, North Carolina \\ 27299 USA $^{4}{ }^{4}$ Department of Molecular Cell Biology, The Weizmann Institute of Science, Rehovot, Israel
}

The p53 gene is the most frequent target of structural and functional genetic mutations in human cancer. Thus, considerable effort has been devoted to mapping the functional domains of p53 with regard to their impact on tumorigenesis in vivo. Studies have shown that the carboxy-terminal domain of p53 is sufficient for transformation in vitro. To determine whether a transdominant-negative $\mathrm{p} 53$ protein could be used to elicit a tissue-specific p53-null effect in vivo, we tested whether a carboxy-terminal p53 fragment (amino acids 302-390) could abolish p53-dependent apoptosis in an established tumor progression model. We showed previously that loss of p53-dependent apoptosis accelerates brain tumorigenesis in a transgenic mouse model. Here, we show that the same effect can be elicited by expressing a dominant-negative p53 protein tissue specifically in the presence of wild-type $\mathrm{p} 53$. Transgenic mice in which $\mathrm{pRb}$ function has been disrupted and that coexpress a 53 carboxy-terminal dominant-negative fragment (p53DD) develop aggressive brain tumors mimicking genetic loss of $\mathrm{p} 53$ in this model. Inactivation of endogenous p53, which we show to be complexed with p53DD, results in a reduction in apoptosis and acceleration of tumorigenesis. These studies establish a mechanism for tissue-specific knock out of $\mathrm{p} 53$ function in vivo.

[Key Words: p53; cancer; apoptosis; transgenic mice; cell specificity]

Received November 7, 1995; revised version accepted February 8, 1996.

Mutation of the p53 gene is the genetic marker most frequently detected in human cancer (Levine et al. 1991; Greenblatt et al. 1994; Hinds and Weinberg 1994). The p53 tumor suppressor functions as a negative regulator of cell proliferation either by its ability to induce growth arrest, programmed cell death or both (Donehower and Bradley 1993; Levine 1993; Picksley and Lane 1994; Haffner and Oren 1995). Based on current evidence, at least two models for the contribution of p53 inactivation to tumorigenesis can be envisioned (Lane 1992; Symonds et al. 1994a; Kastan et al. 1995). In the checkpoint control model, p53-dependent growth arrest or apoptosis in response to DNA damage is lost upon p53 inactivation. Cells that have suffered oncogenic mutations unrelated to 553 survive and divide in the absence of the p53 checkpoint, thereby undergoing the first stage of tumorigenesis. A distinct and more direct role for p53 inactivation in tumor progression is predicted where perturbation of normal cell growth control by oncogenic events precedes p53 mutation. In this case, functional p53 suppresses the survival of emerging tumor cells through p53-dependent apoptosis. Hence, during tumor develop-

Present addresses: ${ }^{2}$ Department of Pharmacology, Duke University, Durham, North Carolina 27708 USA; ${ }^{3}$ Department of Pathology, University of Kentucky, Lexington, Kentucky 40536 USA.

${ }^{5}$ Corresponding author. ment selective pressure exists for the loss of p53 function and p53 inactivation contributes directly to tumor progression. The extent to which either or both of these mechanisms explain the high frequency of p53 inactivation and its role in human tumors is not yet clear. The impact of p53 inactivation may ultimately depend on cell type, stage of cell differentiation, or stage of tumorigenesis in which p53 loss occurs.

Experiments in transgenic mice using the p53-null animals have begun to elucidate the importance of inactivation of p53-dependent apoptosis during tumorigenesis and lend support to this notion. For example, the thymocytes of p53-null mice are defective in DNA damageinduced apoptosis (Clarke et al. 1993; Lowe et al. 1993) and are predisposed to tumorigenesis (Donehower et al. 1992). This result is consistent with loss of a p53-dependent checkpoint contributing to tumor initiation. We reported recently that loss of p53-dependent apoptosis in aberrantly dividing brain epithelial cells results in rapid tumor progression (Symonds et al. 1994b), consistent with the second model described above. Transgenic mice expressing a truncated SV40 large $\mathrm{T}$ antigen that induces abnormal proliferation by interfering with $\mathrm{pRb}$ family functions, but does not inactivate $\mathrm{p} 53$, develop tumors slowly because of abundant p53-dependent apoptosis. However, in a p53-null background, apoptosis is reduced dramatically and the rate of tumorigenesis is accelerated 
(Symonds et al. 1994b). Other laboratories have shown a similar induction of p53-dependent apoptosis in mouse lens (Morgenbesser et al. 1994; Pan and Griep 1994) and retina (Howes et al. 1994), either by using human papilloma virus (HPV) E6 and E7 expression (Pan and Griep 1994), E6 expression in p53 - / - mice (Howes et al. 1994), or $R b-/-p 53-/-$ mice (Morgenbesser et al. 1994).

Further studies are clearly required to determine the cell and developmental-specific mechanisms that define the tumor suppressor functions of p53. However, thus far there is no controlled way to specifically induce p53 loss in somatic cells during specific cell stages in vivo. Although tumor virus proteins such as HPV E6 inactivate p53, these proteins may have other uncharacterized activities, thereby complicating interpretation with respect to loss of p53 function. For example, recent studies show that in the mouse lens E6 interferes with both p53-dependent and p53-independent apoptosis (Pan and Griep 1995).

In vitro studies using dominant mutants of p53 suggest that many of these mutant proteins interfere with wild-type p53 function, inactivating sequence-specific DNA binding and/or transcriptional activation (Shaulian et al. 1992; Unger et al. 1993; Zambetti and Levine 1993). The p53 protein appears to function as a tetramer and can form heteromeric complexes with mutant forms of p53, providing a mechanism for such a dominant-negative action (Milner et al. 1991; Stenger et al. 1992). A region within the carboxy-terminal domain (amino acids 330-390) in murine p53 is responsible for oligomerization (Shaulian et al. 1992; Sturzbecher et al. 1992; Wang et al. 1994). p53 mutant proteins harboring this carboxy-terminal region possess a dominant-negative function and can transform cells in culture, presumably by inactivating endogenous p53 (Unger et al. 1992). Moreover, overexpression of a truncated carboxy-terminal p53 fragment, containing amino acids 302-390, interferes with p53-mediated apoptosis in response to cytokine withdrawal in hematopoietic cells (Gottlieb et al. 1994).

To determine whether tissue-specific expression of a dominant-negative p53 mutant protein could inactivate p53 in vivo, we used a transgenic mouse model of tumorigenesis in which the biological consequences of $\mathrm{p} 53$ inactivation has been established. In the brain epithelial tumor model described above, p53 loss results in a measurable reduction of tumor cell apoptosis and acceleration of tumor progression (Symonds et al. 1994b). This model provides a valid test for controlled somatic inactivation of p53 tumor suppression using a dominant-negative p53 mutant. Here, we have generated transgenic mice that tissue specifically express a p53 mutant harboring only the carboxy-terminal domain p53DD, and we have examined its ability to dominantly inactivate wild-type p53 function in vivo. We report that regulated expression of p53DD elicits tissue-specific inactivation of wild-type p53 tumor suppression in vivo.

\section{Results}

Tissue-specific expression of p53DD in transgenic mice

To test whether the p53DD protein (Fig. 1) could elicit a p53-null effect in tumor progression, we first directed its expression to the choroid plexus epithelium (CPE) of transgenic mice. In this tissue the absence of p53 alone normally has no effect but contributes to rapid tumorigenesis in the abnormally proliferating CPE of $\operatorname{TgT}_{121}$ transgenic mice (Symonds et al. 1994b; summarized in Fig. 2). To achieve CPE expression we used the transcriptional control region of the transthyretin (TTR) gene that is expressed highly in CPE as well as in liver hepatocytes. The transcriptional control elements required for proper tissue-specific expression of TTR-derived constructs have been characterized previously (Costa et al. 1988; Yan et al. 1990; Costa and Grayson 1991). Using transgenic mice, we showed that the TTR promoter targets high-level expression to CPE in a copy number-dependent fashion (Yan et al. 1990). The level of expression in CPE ranges from undetectable with a single copy to $5 \%$ of the total mRNA with 6-10 copies /Yan et al. 1990). Consistently high liver hepatocyte expression is also achieved from the same vector. In this study liver expression provides a control tissue for p53DD effects because liver malignancies in p53-null mice are infrequent (Donehower et al. 1992; Jacks et al. 1994). Moreover, liver expression of p53DD provides an abundant resource for biochemical analyses.

\section{wt p53}
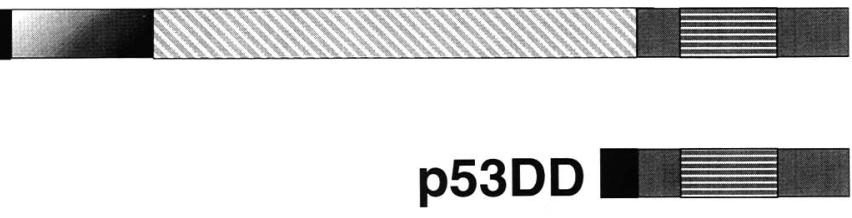

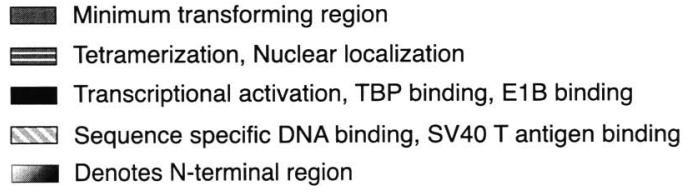

$\square$
$\square$
$\square$ Minimum transforming region Transcriptional activation, TBP binding, E1B binding Denotes $\mathrm{N}$-terminal region
Figure 1. Functional domains of wild-type p53 and p53DD. Functional domains and 100 amino acids regions of protein interaction of are designated by shaded areas. The p53DD cDNA contains the first 13 codons followed by codons 302-390. 


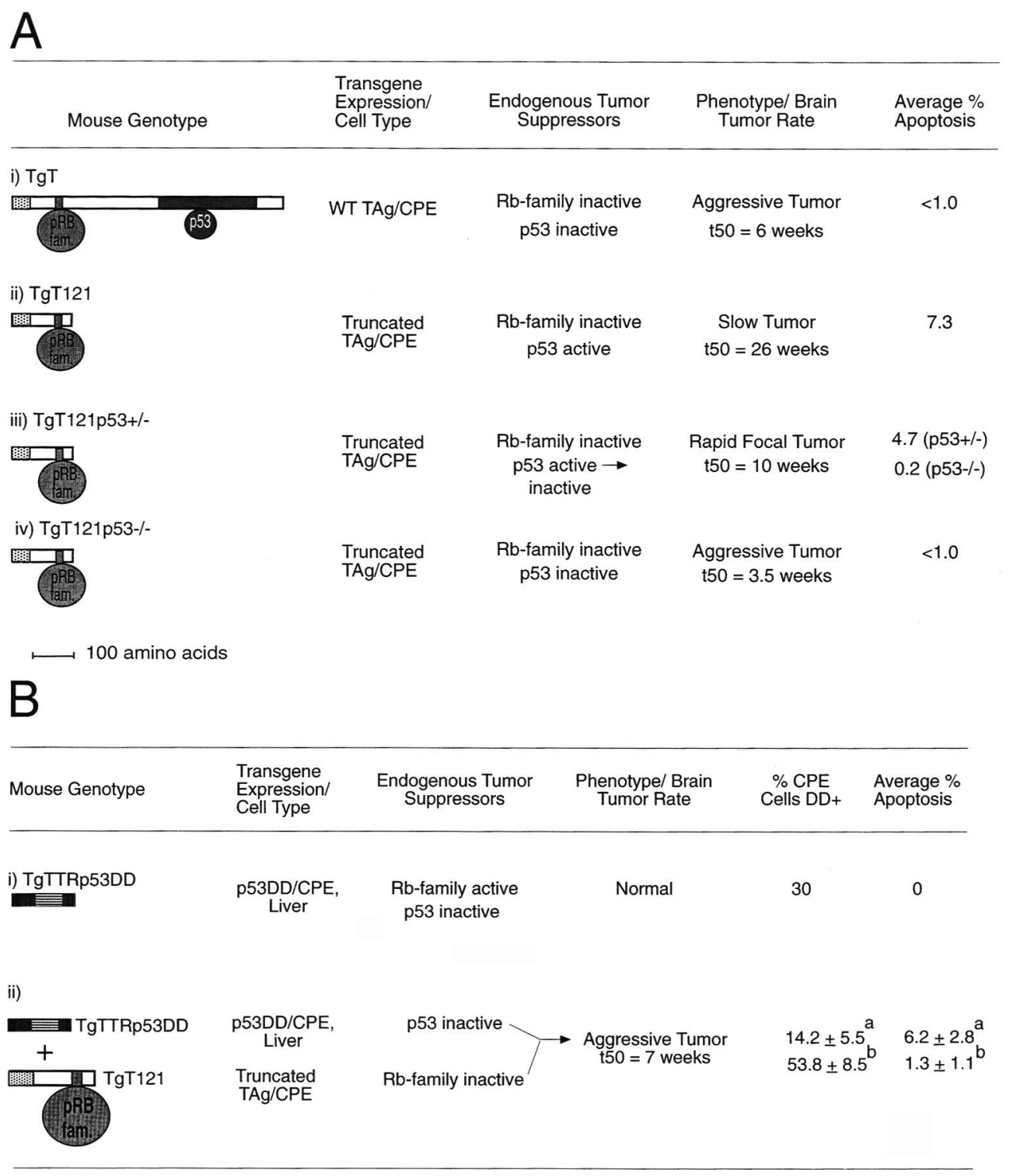

Figure 2. Summary of p53-dependent apoptosis and tumorigenesis. $(A)$ Schematic diagram of wild-type SV40 $\mathrm{T}$ antigen (i) and $\mathrm{T}_{121}$ proteins $(i i-i V)$ with respect to tumorigenic functions. Status of tumor suppressor proteins, type of tumor, rate of tumorigenesis, and average percent apoptotic CPE cells are presented. Average percent apoptosis was quantitated from 10 random fields of vision. $\mathrm{T}_{121}$-induced apoptosis on $\mathrm{p} 53^{+1+}$ and $\mathrm{p} 53^{-1-}$ backgrounds were determined in a previous study (Symonds et al. 1994b). (B) Schematic diagram of p53DD (i)and p53DD and $\mathrm{T}_{121}$ proteins (ii) expressed tissue specifically in CPE and liver. Status of tumor suppressor proteins, tumor kinetics, average percent of total CPE cells expressing the p53DD protein, and average percent apoptosis were determined as indicated in Materials and methods. Two stages of tumor development were analyzed for mice coexpressing p53DD and $\mathrm{T}_{121}$. (a) 22-day-old mouse; $(b)$ 85-day-old mouse.

\section{Analysis of p53DD expression in tissues} of transgenic and nontransgenic littermates

We generated five distinct founder mice harboring the TTRp53DD transgene (TgTTRp53DD-1 through TgTTRp53DD-5|, four of which gave rise to $F_{1}$ offspring. These offspring were analyzed for $\mathrm{p} 53 \mathrm{DD}$ expression in liver and brain by immunoprecipitation analysis using the antibody PAb421, which interacts with the p53 carboxyl terminus (Fig. 3A). Detection of p53DD was then performed by gel electrophoresis and Western blotting. As expected, p53DD was expressed specifically in liver and brain (Fig. 3B), with the level of brain expression varying from line to line (data not shown). All four lines of mice showed abundant p53DD expression in liver. In brain, p53DD was detectable in two lines, TgTTRp53DD-2 and TgTTRp53DD-4. The TgTTRp53DD-2 mice, which expressed the highest level of p53DD protein in brain (data not shown), were used in all subsequent analyses. In some cases (see below), TgTTRp53DD-4 mice were examined as well. 


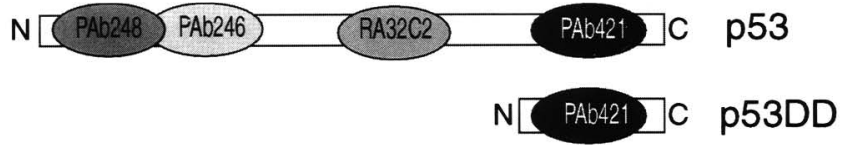

B

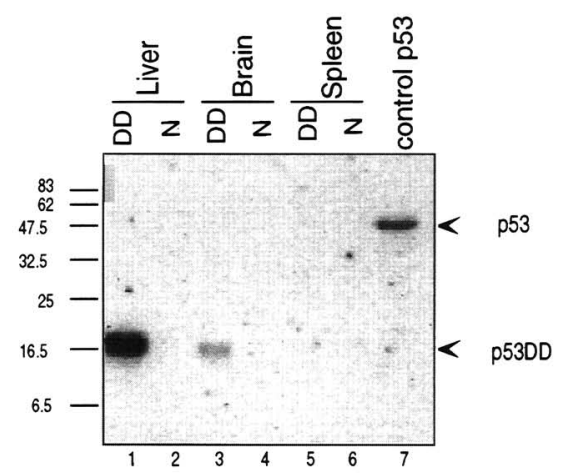

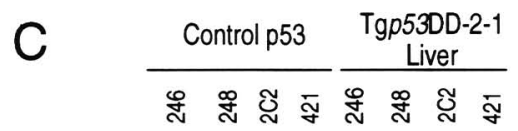

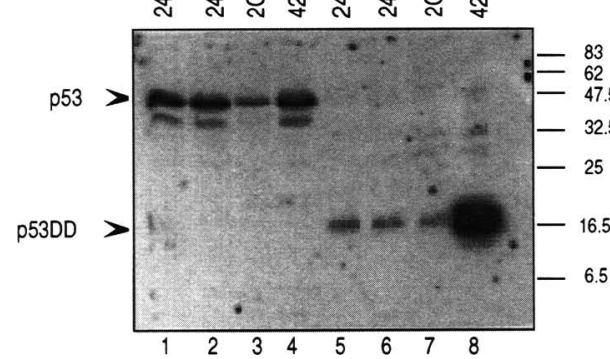

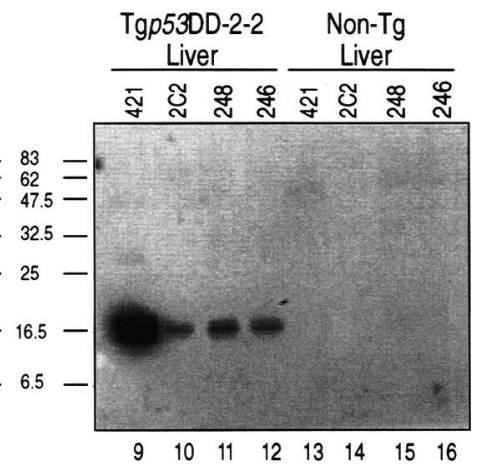

Figure 3. Tissue-specifc expression of p53DD and complex formation with endogenous p53. $(A)$ Schematic diagram of epitope map of wild-type p53 and p53DD indicating regions of recognition for monoclonal antibodies used in $B$ and $C$. (B) Immunoprecipitation-Western analysis for p53DD protein expression in liver, brain, and spleen tissues from a TgTTRp53DD transgenic mouse (DD) and nontransgenic littermate (N). Tumor tissue (control p53) from a transgenic mouse expressing a mutant $T$ antigen (dl1135) that stabilizes p53 was used to detect wild-type p53 (Symonds et al. 1993). Both wild-type and p53DD were immunoprecipitated with mouse monoclonal antibody PAb421, which recognizes an epitope in the carboxyl terminus (see $A$ ), followed by Western blot analysis using PAb421 as the primary antibody. Proteins were detected using ${ }^{125}$ I-labeled protein A. Autoradiographic film was exposed $24 \mathrm{hr}$ with intensifying screens. To achieve antibody excess conditions for liver analysis, $0.1 \mathrm{mg}$ of total protein was used in the immunoprecipitation compared with $2 \mathrm{mg}$ for brain and $5 \mathrm{mg}$ for spleen. For comparison of p53DD levels relative to brain, this represents $\sim 10$-fold more specific cell protein in that hepatocytes account for the majority of liver mass while choroid plexus comprises only $0.5 \%$ of brain mass. (C) Immunoprecipitation-Western analysis for detection of wild-type p53 complexed with p53DD using a battery of mouse monoclonal antibodies specific for epitopes present in wild-type p53 but not p53DD (see A). Control tumor tissue (as in $B$ ) served as a source of wild-type p53 that was immunoprecipitated with each of the indicated mouse monoclonal antibodies (lanes 1-4). Liver extracts from two TgTTRp53DD mice were analyzed for complex formation between wild-type p53 and p53DD (lanes 5-12). Immunoprecipitation of TgTTRp53DD liver extract with a nonspecific monoclonal antibody showed no bands comigrating with either 553 or p53DD (data not shown). Liver extract from a nontransgenic littermate served as a negative control (lanes 13-16). Autoradiographic film was exposed for 1 week with intensifying screens. The positions of wild-type $\mathrm{p} 53, \mathrm{p} 53 \mathrm{DD}$, and molecular mass markers $(\mathrm{kD})$ are indicated.

A representative immunoprecipitation-Western analysis showing p53DD expression in brain and liver is shown in Figure 3B. As expected, no p53DD was detected in transgenic spleen (lane 5) or in any tissue of a nontransgenic littermate (lanes 2,4,6). Tumor tissue from a transgenic mouse expressing SV40 $\mathrm{T}$ antigen, and thus high levels of wild-type p53, served as a control for p53 detection (Fig. 3B, lane 7). Although the endogenous level of p53 in TgTTRp53DD transgenic and nontransgenic tissues was too low to observe in the exposure shown in Figure 3B, longer exposures of the immunoblot showed a weak signal at the position of full-length p53 present to similar levels in all transgenic and nontransgenic tissues (data not shown).

Immunohistochemical analysis of p53 expression was used to confirm the cell-type specificity of p53DD expression. Endogenous p 53 was not detectable under the conditions used (Fig. 4, insets), whereas a strong signal representative of p53DD was observed consistently in transgenic liver hepatocytes and brain $\mathrm{CPE}$ using the carboxy-terminal-specific antibody PAb421 (Fig. 4A,B) but not with RA32C2, a monoclonal antibody that recognizes an epitope in wild-type p53 not present in p53DD (see Fig. 3A; data not shown). Expression of p53DD was distributed uniformly in both liver and choroid plexus, with $\sim 46 \%$ of hepatocytes and $32 \%$ of the brain epithelial cells staining positive (Figs. 4, A and B, respectively).

\section{p53DD forms a complex with endogenous wild-type p53 in vivo}

To determine whether the p53DD expressed in vivo formed a complex with wild-type p53 (as may be predicted for a dominant-negative function), coimmunoprecipitation-Western analysis was performed using a battery of monoclonal antibodies specific for p53 epitopes not present in p53DD (Fig. 3C). Coimmunoprecipitation of p53DD under these conditions indicates a complex 

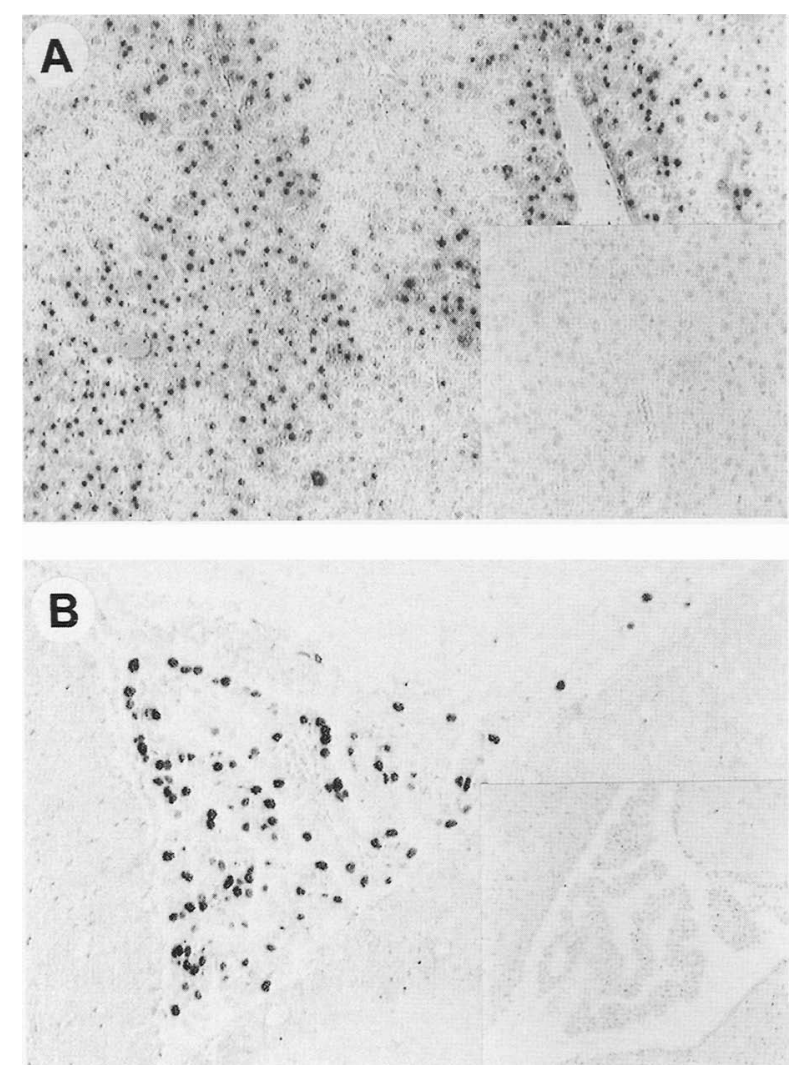

Figure 4. Immunohistochemical staining for $\mathrm{p} 53 \mathrm{DD}$ protein in liver $(A)$ and brain $(B)$ using mouse monoclonal antibody PAb42l. (Insets) In situ staining with the same antibody of tissues from nontransgenic littermates. Expression in liver is limited to hepatocytes and in brain to CPE cells. Magnification, $64 \times$.

between $\mathrm{p} 53 \mathrm{DD}$ and the endogenous wild-type $\mathrm{p} 53$ protein. Lysates from control tumor tissue that abundantly expressed wild-type p53 (lanes 1-4) and transgenic liver tissue from two separate TgTTRp53DD mice (lanes 5-12) were incubated with monoclonal antibodies PAb246, PAb248, or RA32C2, which do not recognize p53DD, or PAb421, which recognizes both wild-type p53 and p53DD (Fig. 3A). Wild-type p53 was readily immunoprecipitated from the control tumor tissue (lanes 14) by all four antibodies. As expected, a much lower level of wild-type p53 was detectable in livers from TgTTRp53DD transgenic (lanes 5-12) and nontransgenic littermates (lanes 13-16). Coimmunoprecipitation of p53DD with antibodies PAb246, PAb248, and RA32C2 (lanes 5-7 and 10-12) indicates the presence of a complex between wild-type and mutant p53 in each p53DDexpressing tissue. Neither p53 nor p53DD were detected using a nonspecific monoclonal antibody for immunoprecipitation (data not shown). The p53DD protein was clearly in excess of wild-type p53, as indicated by total amount of p53DD immunoprecipitated with PAb421 (lanes 8,9 ). As expected, p53DD was not detectable in livers of nontransgenic mice with any antibody (lanes
13-16). These studies show that p53DD forms a complex with wild-type p53 in the tissues of transgenic mice and thus could serve to inactivate wild-type p53 by a dominant-negative mechanism involving complex formation.

\section{Inactivation of $p 53$ by p53DD promotes rapid brain tumor progression}

We showed previously that the $T_{121}$ mutant SV40 T antigen induces abnormal proliferation when expressed in the brain CPE of transgenic mice (Saenz-Robles et al. 1994). This mutant $T$ antigen fragment consists of the amino-terminal 121 amino acid residues of $\mathrm{T}$ antigen (Pipas et al. 1983) and interacts with the pRb family proteins but lacks the p53-binding domain (Fig. 2A, ii-iv). Transgenic mice expressing $T_{121}$ in CPE develop slowgrowing brain tumors, with a $t_{50}=26$ weeks (Figs. 2A, ii, and Fig. 5; Saenz-Robles et al. 1994). The tumors develop slowly because of a high apoptotic index. However, genetic loss of $\mathrm{p} 53$ causes rapid tumor progression $\mid t_{50}=3.5$ weeks) concomitant with a reduction in the apoptotic index (Symonds et al. 1994b; cf. Fig. 2, i, with Fig. 2, iv, and Fig. 5). Thus, assessment of tumor growth rate and

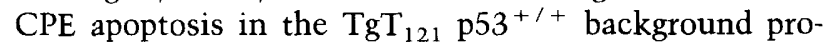
vides a biological assay for $\mathrm{p} 53$ inactivation.

To test the hypothesis that expression of p53DD may elicit a tissue-specific p53-null phenotype, mice of

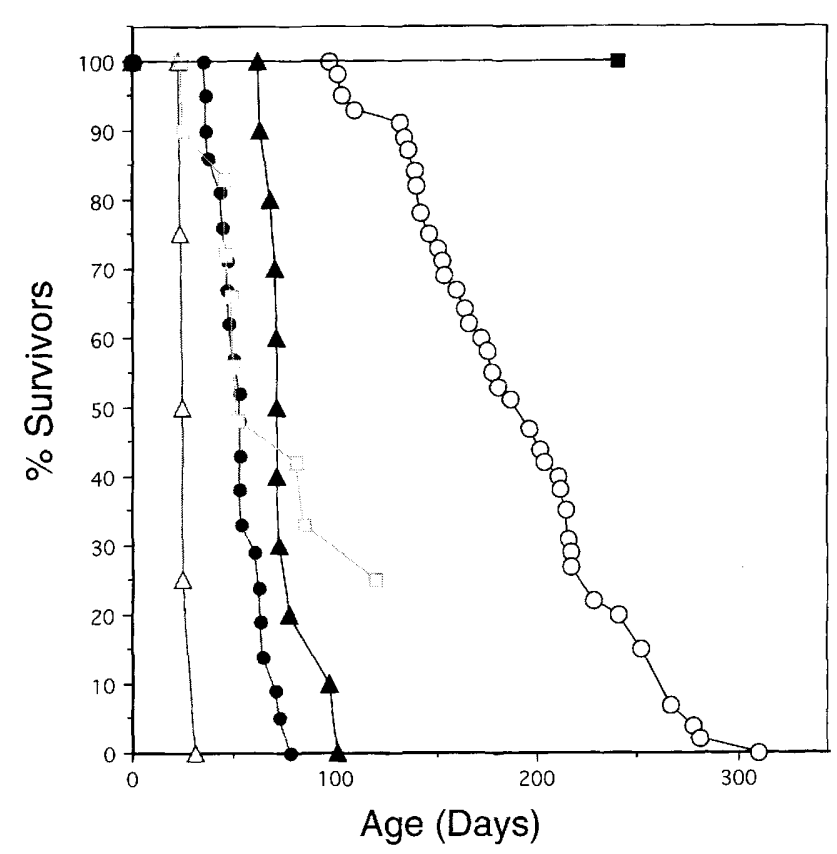

Figure 5. Survival of transgenic mice in relation to p53 function. The percentage of mice of indicated genotypes surviving with time is shown. Survival times reflect the time of sacrifice dictated by the presence of bulged crania that signified the terminal phase of tumorigenesis. Each point on the graph designates an individual mouse. Mouse genotypes are represented by

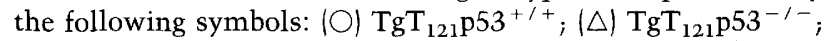

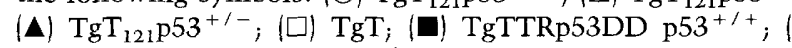
$\operatorname{TgT}_{121} \times$ TgTTRp53DD p53 ${ }^{+1+}$. 
the $\operatorname{TgT}_{121}$ lineage were crossed with mice of the TgTTRp53DD-2 or TgTTRp53DD-4 lineage such that $\mathrm{T}_{121}$ and $\mathrm{p} 53 \mathrm{DD}$ would be coexpressed in the CPE. The wild-type p53 locus is fully intact in these doubly transgenic mice. Expression of p53DD alone had no adverse effect either in liver or in CPE (as is the case for p53-null mice; Donehower et al. 1992; Jacks et al. 1994), and the TgTTRp53DD mice lived a normal life span (Fig. 5). However, coexpression of $\mathrm{T}_{121}$ and $\mathrm{p} 53 \mathrm{DD}$ in the $\mathrm{CPE}$ facilitated rapid tumorigenesis characteristic of $\operatorname{Tg}_{121}$ mice lacking a functional p53 allele (Fig. 2, cf. A and B, and Fig. 5). Mice doubly transgenic for $\mathrm{T}_{121}$ and $\mathrm{p} 53 \mathrm{DD}$ developed CPE tumors with an accelerated rate $\left(t_{50}=7\right.$ weeks) compared with mice expressing $\mathrm{T}_{121}$ alone $\left(t_{50}=26\right.$ weeks $)$ (Fig. 5). The slightly slower rate of tumor growth in $\mathrm{TgT}_{121}$ p53DD-expressing mice compared with p53-null mice $\left(t_{50}=3.5\right.$ weeks $)$ can be explained by the fact that $\mathrm{p} 53 \mathrm{DD}$ was expressed in only $\sim 32 \%$ of the CPE cells (Fig. 4B). In support of this explanation, tumor growth rate in $\mathrm{TgT}_{121} / \mathrm{TgTTRp53DD}$ mice was similar to that of transgenic mice expressing wild-type $\mathrm{T}$ antigen in a similar fraction of CPE cells $\left(t_{50}=6\right.$ weeks; Fig. 5; Chen and Van Dyke 1991). These results indicate that tissue-specific expression of p53DD can functionally inactivate wild-type p53 leading to rapid brain tumor progression.

\section{Expression of p53DD in proliferating CPE correlates with reduced apoptosis}

To determine whether p53DD expression was effective in reducing the p53-dependent apoptosis associated with rapid tumor progression, we analyzed apoptotic indices in CPE cells coexpressing p53DD and $\mathrm{T}_{121}$ or $\mathrm{T}_{121}$ alone. We showed previously that $\mathrm{T}_{121}$ is expressed in a high fraction $(>90 \%)$ of the CPE cells in $\mathrm{TgT}_{121}$ transgenic mice (Saenz-Robles et al. 1994). Figure 6 shows immunohistochemical staining for apoptotic cell nuclei and p53DD protein in brain sections of $T_{121}$ p53DD doubly transgenic mice at two ages. Apoptotic indices relative to that of $\mathrm{T}_{121} \mathrm{p} 53^{+/+}$mice and the percentage of p53DD-expressing cells are presented in the histogram of Figure 6E. Because only a fraction of the CPE cells express p53DD in young mice, we correlated apoptosis with p53DD expression by detecting both events in the same section. Although the apoptotic index was reduced only slightly in young $\mathrm{T}_{121}$ p53DD doubly transgenic mice $\left(85 \%\right.$ that of $T_{121}$ mice; Fig. $\left.6 \mathrm{~A}, \mathrm{~B}, \mathrm{E}\right)$, most apoptotic cells did not express p53DD (Fig. 6B). The apoptotic index was reduced dramatically when, at later times, p53DD-expressing cells became much more abundant (presumably because of their selective advantage) (Fig. 6C-E). The CPE apoptotic indices in four independent $\mathrm{T}_{121} / \mathrm{p} 53 \mathrm{DD}$ mice with a high fraction of $\mathrm{p} 53 \mathrm{DD}-$ positive CPE cells were comparable with that of mice expressing $\mathrm{T}_{121}$ on a genetically p53-null background $(17 \%$, $25 \%, 11 \%$, and $6.5 \%$ compared with $15 \%$ of the $\mathrm{T}_{121}$ control, respectively; Fig. 6E). These results indicate that p53DD expression in the presence of wild-type p53 leads
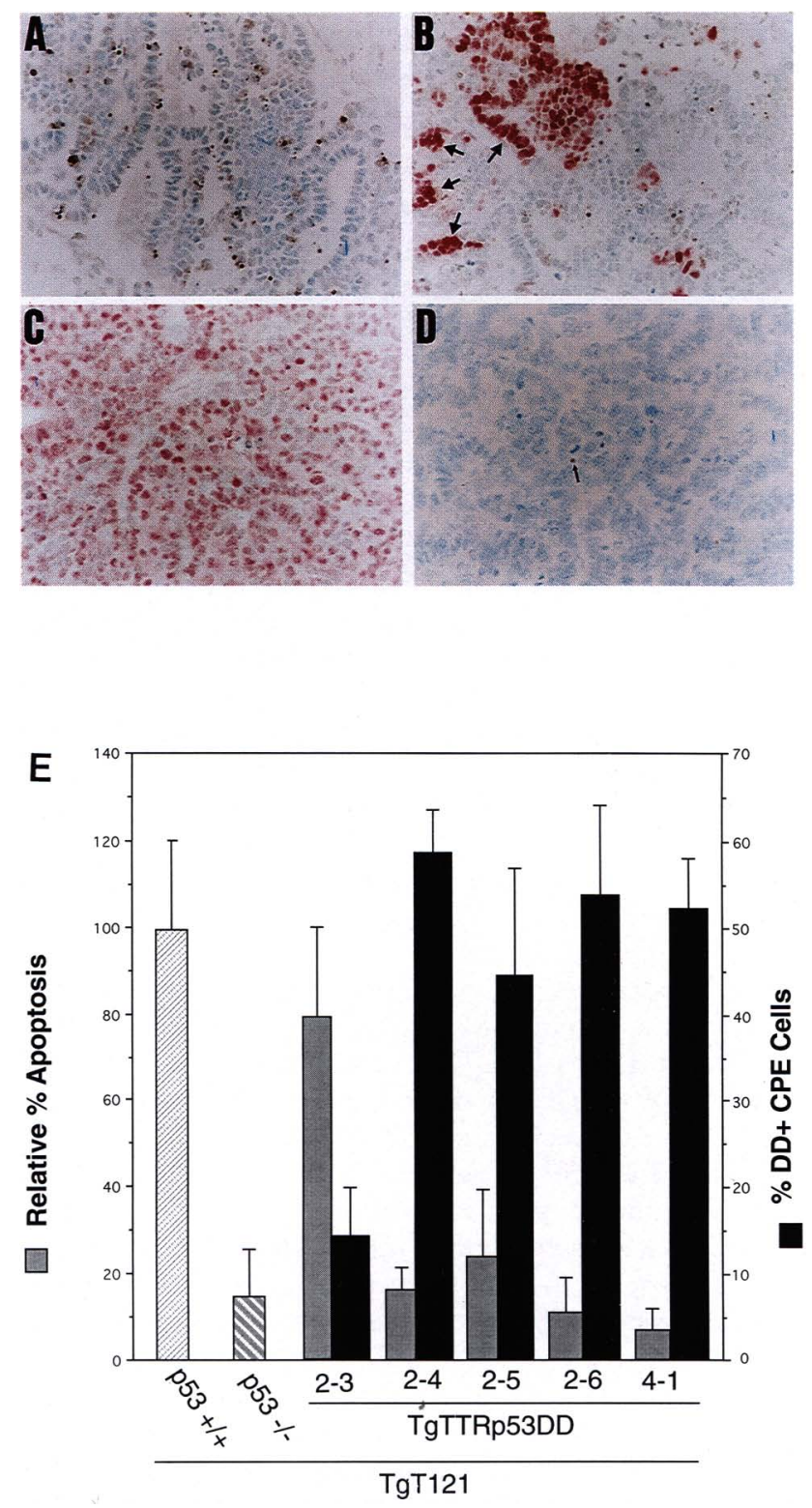

Figure 6. Expression of p53 decreases apoptosis. The CPE of $\mathrm{TgT}_{121} / \mathrm{TTR} 53 \mathrm{DD}$ mice at two different ages was analyzed for cells expressing p53DD protein (using PAb421) and for apoptotic nuclei (using TUNEL) in thin brain tissue sections. (A) Immunohistochemical staining for apoptotic nuclei; (B) p53DD protein simultaneous with apoptotic nuclei in a 22 -day-old mouse. Arrows in $B$ point to regions of CPE cells expressing p53DD protein. $(C)$ In situ detection of p53DD-expressing cells; $(D)$ apoptotic nuclei (as described above) in an 85-day-old $\mathrm{T}_{121}$ TTRp53DD mouse. The arrow in $D$ points to apoptotic nucleus. Magnification in $A-D, 128 \times$. (E) Apoptotic index relative to p53DD expression. Level of apoptosis and number of p53DDexpressing CPE cells were quantified (see Materials and methods) for $\operatorname{TgT}_{121} \mathrm{p} 53^{+/+}, \operatorname{TgT}_{121} \mathrm{p} 53^{-/-}$and five $\operatorname{Tg}_{121} \times \operatorname{TgTTRp53DD}$ mice. Relative percent apoptosis com-

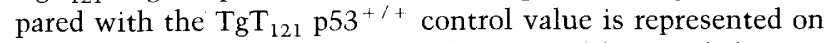
the left $y$-axis corresponding to the stippled bars and the percentage of total CPE cells expressing p53DD is represented on the right $y$-axis corresponding to the solid bars. Data for

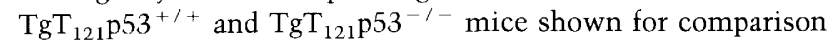
were obtained in a previous study (Symonds et al. 1994b). 
to a reduction in apoptosis consistent with the inactivation of $\mathrm{p} 53$ function by a dominant-negative mechanism.

\section{Discussion}

We have demonstrated here that tissue-specific expression of the p53 carboxy-terminal fragment p53DD, in the presence of wild-type p53, elicits a p53-null phenotype in transgenic mice. The $\mathrm{TgT}_{121}$ tumor model served here as a biological test for p53 inactivation during tumorigenesis in an intact animal model system. These animals, which express the $T_{121}$ truncated SV40 $\mathrm{T}$ antigen specifically in brain epithelium, develop slow growing tumors. The tumor cells proliferate abnormally, a response dependent on the ability of $T_{121}$ to interfere with the function of one or more $\mathrm{pRb}$ family members. In the presence of functional p53, however, these cells undergo p53-dependent apoptosis resulting overall in slow tumor growth. Genetic inactivation of p53 results in a reduction of apoptosis and significant acceleration of tumor growth (Symonds et al. 1994b). This phenotype was reproduced here by expression of $\mathrm{p} 53 \mathrm{DD}$ in $\mathrm{T}_{121}$-expressing brain epithelium in the presence of wild-type p53. Thus, p53DD acts as a dominant-negative inhibitor of p53 function in vivo.

The p53DD transgene was shown to be expressed specifically in brain CPE and in liver hepatocytes by in situ immunohistochemical and immunoprecipitation-Western blot analyses (Figs. 3 and 4, respectively). Overexpression of $\mathrm{p} 53 \mathrm{DD}$ in these cells of otherwise normal mice did not result in abnormalities, and thus the TgTTRp53DD mice have normal life spans. Hence, overexpression of a protein that interferes with p53 function is not toxic under normal circumstances, and p53DD does not appear to possess dominant oncogenic activity distinct from wild-type p53 inactivation. The fact that abnormalities are not induced in hepatocytes and brain epithelium upon inactivation of p53 by p53DD is consistent with observed abnormalities in p53-null and p53-heterozygous mice. p53-null mice do not develop abnormalities in either cell type but, rather, succumb predominantly to $T$-cell lymphomas at $\sim 4-5$ months of age (Donehower et al. 1992; Jacks et al. 1994). The p53 $3^{+-}$mice develop a broader spectrum of tumors than do p53-null mice; however, liver and brain abnormalities have been reported only infrequently (Jacks et al. 1994; Harvey et al. 1995).

Although p53DD had no impact on normal nondividing brain epithelial cells, its presence accelerated tumor development significantly when coexpressed with the $T_{121} T$ antigen fragment. As with genetic inactivation of p53, the acceleration of tumor growth correlated with a reduction in apoptosis. Because only a fraction of CPE cells $(\sim 32 \%)$ expressed p53DD, tumors developed at a slower rate compared with that of $\mathrm{T}_{121} \mathrm{p} 53^{-1-}$ mice $\left(t_{50}=7\right.$ weeks vs. 3.5 weeks, respectively) but accelerated significantly compared with $\mathrm{TgT}_{121} \mathrm{p} 53^{+/+}$mice $\left(t_{50}=26\right.$ weeks). The fact that $553 \mathrm{DD}$ may not be expressed in all cells of a given tissue when introduced as a transgene provides an experimental advantage, as cells that are functionally $\mathrm{p} 53^{+}$and $\mathrm{p} 53^{-}$can be assessed in the same tissue. This situation reflects more accurately what occurs in a developing neoplasm upon somatic inactivation than does a genetic null in which the gene is never expressed. In the case of the doubly transgenic $\mathrm{T}_{121}$ / TTRp53DD mice, CPE cells that expressed p53DD had a clear growth advantage over those expressing $T_{121}$ alone. This was apparent upon examining the CPE at different ages where multifocal tumor growths were consistently p53DD positive. Moreover, there was a correlation between the fraction of cells expressing p53DD and a reduction in apoptotic index, with predominantly nonexpressing cells undergoing apoptosis before focal growth. This observation supports the previous indication that the selective growth advantage is a reduction in cell death.

The mechanism of $\mathrm{p} 53$ inactivation by $\mathrm{p} 53 \mathrm{DD}$ likely involves hetero-oligomerization with the endogenous p53. p53 binds specific DNA optimally as a tetramer (Shaulian et al. 1992; Unger et al. 1993; Wang et al. 1994), and wild-type p53 is capable of oligomerization in normal tissues (Wang et al. 1994). Previous studies in tissue culture have shown that p53DD interacts with wild-type p53 and also interferes with p53 DNA-binding activity (Shaulian et al. 1992, 1995). In this study we showed that p53DD can dominantly inactivate the tumor suppression function of p53. Furthermore, we demonstrated that $\mathrm{p} 53 \mathrm{DD}$ forms a complex with wild-type p53 in vivo (Fig. 3). Using antibodies specific for the fulllength protein but that did not interact with the mutant protein directly, p53DD was readily coimmunoprecipitated from tissue extracts with wild-type p53. Most if not all of the endogenous p53 in p53DD-expressing cells was likely in such complexes because p53DD was present in vast excess (Fig. $3 \mathrm{C}$ ).

Our studies show compellingly that tissue-specific expression of $\mathrm{p} 53 \mathrm{DD}$ can be used as a valuable tool to study a variety of tumors at various developmental stages in existing mouse models. Although p53 is the gene affected most frequently in human cancer, it is not clear whether a common mechanism associated with p53 loss contributes to the development of tumors of different cell origins. Mutations in p53 arise in most if not all histogenic classes of tumors (Hollstein et al. 1991). In some cases, p53 mutation can be detected early in the development of malignancy, whereas such mutations consistently appear in other cancer types later in progression (for review, see Hollstein et al. 1991). The consequences of p53 loss may be different, depending on the stage of tumorigenesis at which p53 inactivation occurs. For example, $\mathrm{p} 53^{-1-}$ and $\mathrm{p} 53^{+/-}$mice develop a different spectrum of tumors for currently unknown reasons (Harvey et al. 1993; Jacks et al. 1994) Although mouse models have contributed significantly to our understanding of p53 function, currently we are limited to studying the complete absence of p53 (as in a p53-null genetic background) or stochastic loss of p53 (as in p53-heterozygous mice). The latter situation is most similar to what occurs in the development of human tumors but is not regulatable experimentally. Our studies on p53DD 
offer a third, more manipulable system for studying p 53 loss during specific stages of tumorigenesis in multiple cell types and provide an alternative to the use of viral proteins, such as HPV E6. In this study targeted inactivation of $\mathrm{p} 53$ was accomplished using a cell-specific promoter to drive p53DD expression. Additional versatility could be accomplished using an inducible promoter system or with somatic delivery of viral vectors expressing p53DD. Because we have shown that p53DD elicits a biological effect identical to genetic loss of p53, these studies should provide a tool for probing cell- and stagespecific mechanisms of p53 loss in the contribution to tumorigenesis.

\section{Materials and methods}

\section{Plasmids}

The construction of the expression vector containing the truncated $\mathrm{T}$ antigen gene $\mathrm{pT}_{121}$ (formerly pLST1137) was described previously (Chen and Van Dyke 1991; Chen et al. 1992). The vector used for expression of p53DD (TTR1ExV3) has been described (Wu et al. 1996). It contains transcriptional control sequences from the mouse TTR gene required for efficient and tissue-specific expression in the brain CPE and liver. Site-directed point mutagenesis was used to eliminate the ATG codons in the first and second exons of TTR and to provide a StuI restriction site for cDNA insertion in the second exon. The TTRp53DD plasmid was constructed by digesting the expression plasmid pTTRExV3 with StuI followed by the generation of blunt ends with Klenow. The pSPDD described previously (Shaulian et al. 1992) was digested with BamHI and XbaI and blunted with Klenow. The 650-bp coding fragment was isolated and then ligated into pTTRExV3. The construct pTTRp53DD was confirmed by sequence analysis. The resulting p53DD-coding segment contains the murine $\mathrm{p} 53$ initiation codon and the amino-terminal 13 codons followed by carboxy-terminal codons 302-390 (Fig. 1).

\section{Production and screening of transgenic mice}

The plasmid pTTR-p53DD was digested with HindIII, and the 4.7-kb fragment containing the TTRp53DD gene was isolated and microinjected into fertilized mouse eggs $(\mathrm{B} 6 \mathrm{D} 2 \mathrm{~F} 1 \times \mathrm{B} 6 \mathrm{D} 2 \mathrm{~F} 1$; Jackson Laboratories, Bar Harbor, ME) as described previously (Chen and Van Dyke 1991; Chen et al. 1992). Transgenic offspring were identified by PCR analysis of genomic DNA isolated from tail samples using two sets of primers, one that hybridizes to sequences within the TTR gene promoter region and detects the endogenous TTR gene (internal control) as well as the transgene $15^{\prime}$-TCGCGAATTCGGAGCTTGTGGATCTGTGTGACGGC- $3^{\prime}$ and 5'-CTCGAGATCTGGGATTGGGTGTGTC-3') and a transgene-specific primer set that hybridizes to the TTR intron and to p53DD $15^{\prime}$-CTTTTTGCACCATGCACCTTTC- $3^{\prime}$ and 5'-TTGGTCTTCAGGTAGCTGGAGTG$3^{\prime}$, respectively). Generation, screening, and characterization of $\operatorname{Tg} T_{121}$ mice were described previously (Saenz-Robles et al. 1994).

\section{Immunoprecipitation and immunoblotting analysis}

Tissues were used fresh or stored frozen at $-80^{\circ} \mathrm{C}$ before analysis. Extracts were prepared as described previously (Symonds et al. 1993). The protein extract containing $5 \mathrm{mg}$ of total protein from spleen, $2 \mathrm{mg}$ from brain, $0.1 \mathrm{mg}$ from liver, and $0.5 \mathrm{mg}$ from thymus was precleared with Staphylococcus aureus-protein A followed by incubation with the appropriate monoclonal antibody $\left(300 \mu \mathrm{l}\right.$ of hybridoma supernatant) for $1 \mathrm{hr}$ at $4^{\circ} \mathrm{C}$. The antibody-protein complexes were immunoprecipitated with $S$. aureus, the immunoprecipitates washed three times with SNNTE [ $5 \%$ sucrose, $0.5 \mathrm{~mm} \mathrm{NaCl}, 0.1 \% \mathrm{NP}-40,50 \mathrm{~mm}$ Tris (pH 7.4), and 5 mM EDTA] followed by one wash with NTE [50 $\mathrm{mm} \mathrm{NaCl}, 10 \mathrm{~mm}$ Tris (pH 7.4), and $1 \mathrm{~mm}$ EDTA], resuspended in Laemmli protein loading buffer, and electrophoresed in 15\% polyacrylamide-SDS gels. Proteins were transferred to nitrocellulose (BioBlot-NC, Costar), and immunoblotting was performed as described previously using ${ }^{125} \mathrm{I}$-labeled protein $\mathrm{A}$ detection (Symonds et al. 1993). To detect p53/p53DD complexes, liver lysate containing $5 \mathrm{mg}$ of total protein was used for each immunoprecipitation. The following antibodies were used for immunoprecipitation: PAb421 (Harlow et al. 1981), RA32C2 (Coffmann and Weissman 1981), PAb246, and PAb248 (Wade Evans and Jenkins 1985). The approximate locations of epitopes are shown in Figure 3A. A monoclonal antibody specific for adeno-associated virus capsid proteins (provided by R. Samulski, University of North Carolina, Chapel Hill) was used as a nonspecific control for p53 immunoprecipitation.

\section{Histology and immunohistochemistry}

Brain tissues were fixed in $10 \%$ neutral-buffered formalin (Sigma) for 16-20 hr, washed in $\mathrm{dH}_{2} \mathrm{O}$ followed by $70 \%$ ethanol, and embedded in paraffin. Tissue sections $(6 \mu)$ were deparaffinized by successive washes in Hemo-De (Fischer) followed by dehydration in graded ethanols. For immunostaining, deparaffinized sections were incubated with trypsin $(1 \mathrm{mg} / \mathrm{ml})$ in phosphate-buffered saline (PBS) for $30 \mathrm{~min}$ at room temperature. Sections were blocked with $5 \%$ normal goat serum (NGS) in PBS for $30 \mathrm{~min}$ at room temperature, by incubation with primary antibody in 5\% NGS in PBS for $1 \mathrm{hr}$ at room temperature. A mouse monoclonal antibody to p53 that recognizes amino acids 370-378 in the carboxyl terminus (PAb421; Harlow et al. 1981) was used to detect p53DD. The slides were washed in PBS and incubated with biotinylated anti-mouse IgG /diluted 1:300 to 1:500 with NGS in PBS| for $30 \mathrm{~min}$ at room temperature. Immunocomplexes were detected using the Vectastain $\mathrm{ABC}$ alkaline phosphatase kit (Vector) according to the manufacturer's instructions.

In situ detection of apoptosis was performed on tissue sections alone or after immunohistochemical staining. Endogenous peroxidase activity was quenched with $0.1 \% \mathrm{H}_{2} \mathrm{O}_{2}$ in PBS. Apoptotic cells were detected using the in situ Apoptag kit (Oncor) according to the manufacturer's protocol except that the slides were incubated with terminal deoxytransferase for 15 min at room temperature as described previously (Symonds et al. 1994b). Quantification was performed by counting 10 randomly chosen fields of vision at a magnification of $400 \times$ per tissue section. The counts were averaged to obtain the average number of cells expressing p53DD or the average apoptotic index.

\section{Acknowledgments}

We acknowledge Mayté Saenz-Robles and Suzanne Bertera for the production of founder mice and Laura Chadwick for initial crosses. We thank Kim Berns and Tracey Bartolotta of the University of North Carolina Pulmonary histology core facility for histological sample preparation. We also thank John Kim for excellent technical assistance and Stacy Bridge and Lisa Moore for assistance in manuscript preparation. This work is sup- 
ported by grants from the National Institutes of Health (CA46283 and DK42910 to T.V.D. and RO1 CA40099 to M.O.). T.V.D. is the recipient of a research career development award from the National Cancer Institute.

The publication costs of this article were defrayed in part by payment of page charges. This article must therefore be hereby marked "advertisement" in accordance with 18 USC section 1734 solely to indicate this fact.

\section{References}

Chen, J. and T. Van Dyke. 1991. Uniform cell-autonomous tumorigenesis of the choroid plexus by papovavirus large $\mathrm{T}$ antigens. Mol. Cell. Biol. 11: 5968-5976.

Chen, J., G. Tobin, J.M. Pipas, and T.A. Van Dyke. 1992. T Antigen mutant activities in transgenic mice: Roles of p53 and $\mathrm{pRB}$-binding in tumorigenesis of the choroid plexus. Oncogene 7: 1167-1175.

Clarke, A.R., C.A. Purdie, D.J. Harrison, R.G. Morris, C.C. Bird, M.L. Hooper, and A.H. Wyllie. 1993. Thymocyte apoptosis induced by p53-dependent and independent pathways. $\mathrm{Na}$ ture 362: 849-852.

Coffmann, R.L. and I.L. Weissman. 1981. A monoclonal antibody that recognizes B cells and B cell precursors in mice. $J$. Exp. Med. 153: 269-279.

Costa, R.H. and D.R. Grayson. 1991. Site-directed mutagenesis of hepatocyte nuclear factor (HNF) binding sites in the mouse transthyretin (TTR) promoter reveal synergistic interactions with its enhancer region. Nucleic Acids Res. 19: 4139-4145.

Costa, R.H., D.R. Grayson, K.G. Xanthopoulos, and J.E. Darnell. 1988. A liver-specific DNA-binding protein recognizes multiple nucleotide sites in regulatory regions of transthyretin, alpha 1-antitrypsin, albumin, and simian virus 40 genes. Proc. Natl. Acad. Sci. 85: 3840-3844.

Donehower, L.A. and A. Bradley. 1993. The tumor suppressor p53. Biochem Biophys. Acta 1155: 181-205.

Donehower, L.A., M. Harvey, B.L. Slagle, M.J. McArthur, C.A.J. Montgomery, J.S. Butel, and A. Bradley. 1992. Mice deficient for p53 are developmentally normal but susceptible to spontaneous tumours. Nature 356: 215-221.

Gottlieb, E., R. Haffner, T. von Ruden, E. Wagner, and M. Oren. 1994. Down-regulation of wild-type p53 activity interfers with apoptosis of IL-3-dependent hematopoietic cells following IL-3 withdrawal. EMBO J. 13: 1368-1374.

Greenblatt, M.S., W.P. Bennett, M. Hollstein, and C.C. Harris. 1994. Mutations in the p53 tumor suppressor gene: Clues to cancer etiology and molecular pathogenesis. Cancer Res. 54: 4855-4878.

Haffner, R. and M. Oren. 1995. Biochemical properties and biological effects of p53. Curr. Opin. Genet. Dev. 5(1): 84-94.

Harlow, E., L.V. Crawford, D.C. Pim, and N.M. Williamson. 1981. Monoclonal antibodies specific for simian virus 40 tumor antigens. I. Virol. 39: 861-869.

Harvey, M., M.J. McArthur, C.A. Montgomery, J.S. Butel, A. Bradley, and L.A. Donehower. 1993. Spontaneous and carcinogen-induced tumorigenesis in p53-deficient mice. $\mathrm{Na}$ ture Genet. 5(3): 225-229.

Harvey, M., H. Vogel, D. Morris, A. Bradley, A. Bernstein, and L.A. Donehower. 1995. A mutant p53 transgene accelerates tumour development in heterozygous but not nullizygous p53-deficient mice. Nature Genet. 9: 305-311.

Hinds, P.W. and R.A. Weinberg. 1994. Tumor suppressor genes. Curr. Biol. 4: 135-141.

Hollstein, M., D. Sidransky, B. Vogelstein, and C.C. Harris.
1991. p53 mutations in human cancers. Science 253: 49-53.

Howes, K.A., N. Ransom, D.S. Papermaster, J.G.H. Lasudry, D.M. Albert, and J.J. Windle. 1994. Apoptosis or retinoblastoma: alternative fates of photoreceptors expressing the HPV-16 E7 gene in the presence or absence of p53. Genes \& Dev. 8: 1300-1310.

Jacks, T., L. Remington, B. Williams, E. Scmitt, S. Halachmi, R. Bronson, and R. Weinberg. 1994. Tumor spectrum analysis in p53-mutant mice. Curr. Biol. 4: 1-7.

Kastan, M.B., C.E. Canman, and C.J. Leonard. 1995. P53, cell cycle control and apoptosis: Implications for cancer. Cancer Metastasis Rev. 14: 3-15.

Lane, D.P. 1992. p53, guardian of the genome. Nature 358: 1516.

Levine A.J. 1993. The tumor suppressor genes. Annu. Rev. Biochem. 62: 623-651.

Levine, A.J., J. Momand, and C.A. Finlay. 1991. The p53 tumour suppressor gene. Nature 351: 453-456.

Lowe, S.W., E.M. Schmitt, S.W. Smith, B.A. Osborne, and T. Jacks. 1993. p53 is required for radiation-induced apoptosis in mouse thymocytes. Nature 362: 847--849.

Milner, J., E.A. Medcalf, and A.C. Cook. 1991. The tumour suppressor p53: Analysis of wild type and mutant p53 complexes. Mol. Cell. Biol. 11: 12-19.

Morgenbesser, S., B. Williams, T. Jacks, and R. DePinho. 1994. p53-dependent apoptosis produced by Rb-deficiency in the developing mouse lens. Nature 371: 72-74.

Pan, H. and A.E. Griep. 1994. Altered cell cycle regulation in the lens of HPV-16 E6 or E7 transgenic mice: Implications for tumor suppressor gene function in development. Genes \& Dev. 8: 1285-1299.

—_ 1995. Temporally distinct patterns of p53-dependent and p53-independent apoptosis during mouse lens development. Genes \& Dev. 9: 2157-2169.

Picksley, S.M. and D.P. Lane. 1994. p53 and Rb: Their cellular roles. Curr. Opin. Cell Biol. 6(6): 853-858.

Pipas, J.M., K.W. Peden, and D. Nathans. 1983. Mutational analysis of simian virus $40 \mathrm{~T}$ antigen: Isolation and characterization of mutants with deletions in the T-antigen gene. Mol. Cell. Biol. 3: 203-213.

Saenz-Robles, M.T., H. Symonds, J. Chen, and T. Van Dyke. 1994. Induction versus progression of brain tumor development: Differential functions for the pRB- and p53-targeting domains of simian virus $40 \mathrm{~T}$ antigen. Mol. Cell. Biol. 14(4): 2686-2698.

Shaulian, E., A. Zauberman, D. Ginsberg, and M. Oren. 1992. Identification of a minimal transforming domain of p53: Negative dominance through abrogation of sequence-specific DNA binding. Mol. Cell. Biol. 12: 5581-5592.

Shaulian, E., I. Haviv, Y. Shaul, and M. Oren. 1995. Transcriptional repression by the $\mathrm{C}$-terminal domain of $\mathrm{p} 53$. Oncogene 10(4): 671-680.

Stenger, J.E., G.A. Mayr, K. Mann, and P. Tegtmeyer. 1992. Formation of stable p53 homotetramers and multiples of tetramers. Mol. Carcinog. 5: 102-106.

Sturzbecher, H.W., R. Brain, C. Addison, K. Rudge, M. Remm, M. Grimaldi, E. Keenan, and J.R. Jenkins. 1992. A C-terminal alpha-helix plus basic region motif is the major structural determinant of p53 tetramerization. Oncogene 7(8): 15131523.

Symonds, H.S., S.A. McCarthy, J. Chen, J.M. Pipas, and T. Van Dyke. 1993. Use of transgenic mice reveals cell-specific transformation by a simian virus $40 \mathrm{~T}$-antigen amino-terminal mutant. Mol. Cell. Biol. 13: 3255-3265.

Symonds, H., L. Krall, L. Remington, M. Saenz Robles, T. Jacks, and T. Van Dyke. 1994a. p53-Dependent apoptosis in vivo: 
Impact of $\mathrm{p} 53$ inactivation on tumorigenesis. Cold Spring Harbor Symp. Quant. Biol. 59: 247-257.

Symonds, H., L. Krall, L. Remington, M. Saenz-Robles, S. Lowe, T. Jacks, and T. Van Dyke. 1994b. p53-Dependent apoptosis suppresses tumor growth and progression in vivo. Cell 78: 703-711.

Unger, T., M.M. Nau, S. Segal, and J.D. Minna. 1992. p53: A transdominant regulator of transcription whose function is ablated by mutations occurring in human cancer. EMBO $\mathrm{J}$. 11: $1383-1390$.

Unger, T., J.A. Mietz, M. Scheffner, C.L. Yee, and P.M. Howley. 1993. Functional domains of wild-type and mutant proteins involved in transcriptonal regulation, transdominant inhibition, and transformation suppression. Mol. Cell Biol. 13: 5186-5194.

Wade Evans, A. and J.R. Jenkins. 1985. Precise epitope mapping of the murine transformation-associated protein, p53. EMBO I. 4: 699-706.

Wang, P., M. Reed, Y. Wang, G. Mayr, J.E. Stenger, M.E. Anderson, J.F. Schwedes, and P. Tegtmeyer. 1994. p53 domains: Structure, oligomerization, and transformation. Mol. Cell. Biol. 14(8): 5182-5191.

Wu, H., M. Wade, L. Krall, J. Grisham, Y. Xiong, and T. Van Dyke. 1996. Targeted in vivo expression of the cyclin-dependent kinase inhibitor p21, halts hepatocyte cell cycle progression, postnatal liver development, and regeneration. Genes \& Dev. 10: 245-260.

Yan, C., R.H. Costa, J.E. Darnell, J.D. Chen, and T.A. Van Dyke. 1990. Distinct positive and negative elements control the limited hepatocyte and choroid plexus expression of transthyretin in transgenic mice. EMBO J. 9: 869-878.

Zambetti, G. and A. Levine. 1993. A comparison of the biological activities of wild-type and mutant p53. FASEB I. 7: 855865. 


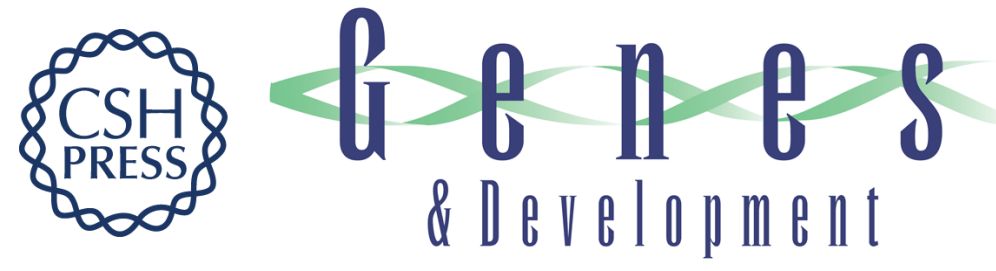

\section{Tissue-specific inactivation of p53 tumor suppression in the mouse.}

T Bowman, $\mathrm{H}$ Symonds, L Gu, et al.

Genes Dev. 1996, 10:

Access the most recent version at doi:10.1101/gad.10.7.826 $\begin{array}{ll}\text { References } & \begin{array}{l}\text { This article cites } 44 \text { articles, } 18 \text { of which can be accessed free at: } \\ \text { http://genesdev.cshlp.org/content/10/7/826.full.html\#ref-list-1 }\end{array}\end{array}$

License

Email Alerting

Receive free email alerts when new articles cite this article - sign up in the box at the top Service right corner of the article or click here.

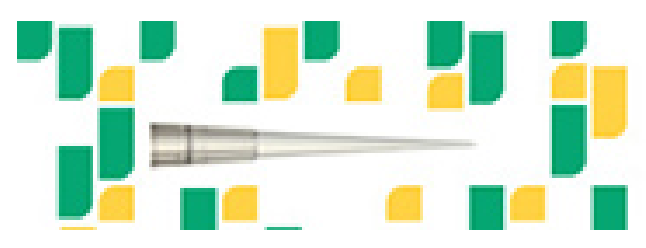

Focused on your science.

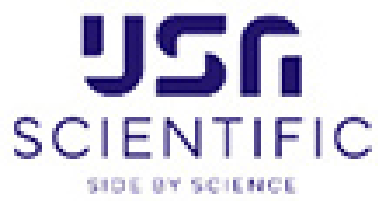

Copyright (c) Cold Spring Harbor Laboratory Press 